\title{
NEW PERSPECTIVES IN TURBULENCE
}

\author{
$\mathrm{BY}$ \\ ALEXANDRE J. CHORIN \\ Department of Mathematics, University of California, Berkeley, California
}

\begin{abstract}
An analysis of the mean velocity profile in the intermediate region of wall-bounded turbulence shows that the well-known von Kármán-Prandtl logarithmic law of the wall must be jettisoned in favor of a power law. An analogous analysis of the local structure of turbulence shows that the Kolmogorov-Obukhov scaling of the second and third structure functions is exact in the limit of vanishing viscosity while, in the same limit, higher-order moments fail to exist. These results rely on advanced similarity methods and on vanishing-viscosity asymptotics, and are consistent with a near-equilibrium theory of turbulence of which a new version is presented.
\end{abstract}

1. Introduction. In February 1996 I had the privilege of meeting Prof. G. I. Barenblatt, who had just arrived in Berkeley. In our first extended conversation we discovered that we had been working on similar problems with different but complementary tools, which, when wielded in unison, let to unexpected results. We have been working together ever since, and it is a pleasure to be able to present some of the results of our joint work at this distinguished occasion.

The present talk will consist of three parts: (i) an application of advanced similarity methods and vanishing-viscosity asymptotics to the analysis of wall-bounded turbulence, (ii) a discussion of the local structure of turbulence with particular attention to the higher-order structure functions, and (iii) a discussion of a near-equilibrium statistical theory of turbulence, which motivates and complements our reading of the numerical and experimental data. The basic premise is that, as the viscosity tends to zero and the solutions of the Navier-Stokes equations acquire poorly understood temporal and spatial fluctuations, certain mean properties of the flow can be seen to take on well-defined limits, which can be found by expansion in a small parameter that tends to zero, albeit slowly, as the viscosity tends to zero.

In the case of wall-bounded turbulence, our argument and the data show that the classical von Kármán-Prandtl law should be replaced, when the viscosity is small but

Received May 29, 1997.

1991 Mathematics Subject Classification. Primary 76F10, 76F05, 76C05, 82C99.

Supported in part by the Applied Mathematical Sciences subprogram of the Office of Energy Research, U. S. Department of Energy, under contract DE-AC03-76-SF00098, and in part by the National Science Foundation under grants DMS94-14631 and DMS89-19074. 
finite, by a Reynolds-number-dependent power law. In the case of local structure, an analogous argument shows that the Kolmogorov scaling of the second and third-order structure functions is exact in the limit of vanishing viscosity, when the turbulence is most intermittent and least organized. When the viscosity is nonzero (Reynolds number large but finite), Reynolds-number-dependent corrections to the Kolmogorov-Obukhov scaling of the structure functions appear, due to a viscosity-induced reduction in intermittency. For higher-order structure functions the vanishing viscosity limit ceases to exist because of intermittency, and the Kolmogorov-Obukhov scaling fails.

The near-equilibrium statistical theory we shall present is the basis of vanishingviscosity asymptotics and relates the behavior of the higher-order structure functions to the presence of intermittency. All parts of our analysis are heterodox in the context of the current state of turbulence research, but not in the broader context of the statistical mechanics of irreversible phenomena.

2. The intermediate region in wall-bounded turbulence. Consider wallbounded turbulence, in particular, fully-developed turbulence in the working section, i.e., far from the inlet and outlet, of a long cylindrical pipe with circular cross section. It is customary to represent $u$, the time-averaged or ensemble-averaged longitudinal velocity in a pipe in the dimensionless form

$$
\phi=u / u_{*}
$$

where $u_{*}$ is the "friction" velocity that defines the velocity scale:

$$
u_{*}=\sqrt{\tau / \rho},
$$

where $\rho$ is the density of the fluid and $\tau$ is the shear stress at the pipe's wall,

$$
\tau=\frac{\Delta p}{L} \frac{d}{4}
$$

Here $\Delta p$ is the pressure drop over the working section of the pipe, $L$ is the length of the working section, and $d$ is the pipe's diameter. The dimensionless distance from the pipe wall is

$$
\eta=\frac{u_{*} y}{\nu}
$$

where $y$ is the actual distance from the wall and $\nu$ is the fluid's kinematic viscosity. The length scale $\nu / u_{*}$ in (2.4) is typically very small-less than tens of microns in some of the data discussed below. The key dimensionless parameter in the problem is the Reynolds number

$$
R e=\frac{\bar{u} d}{\nu}
$$

where $\bar{u}$ is the velocity averaged over the cross section. When the Reynolds number $R e$ is large, one observes that the cross section is divided into three parts (Fig. 1): (1) the viscous sublayer near the wall, where the velocity gradient is so large that the shear stress due to molecular viscosity is comparable to the turbulent shear stress, a cylinder (2) surrounding the pipe's axis where the velocity gradient is small and the average velocity is close to its maximum, and the intermediate region (3) which occupies most of the cross section and on which we shall focus. 


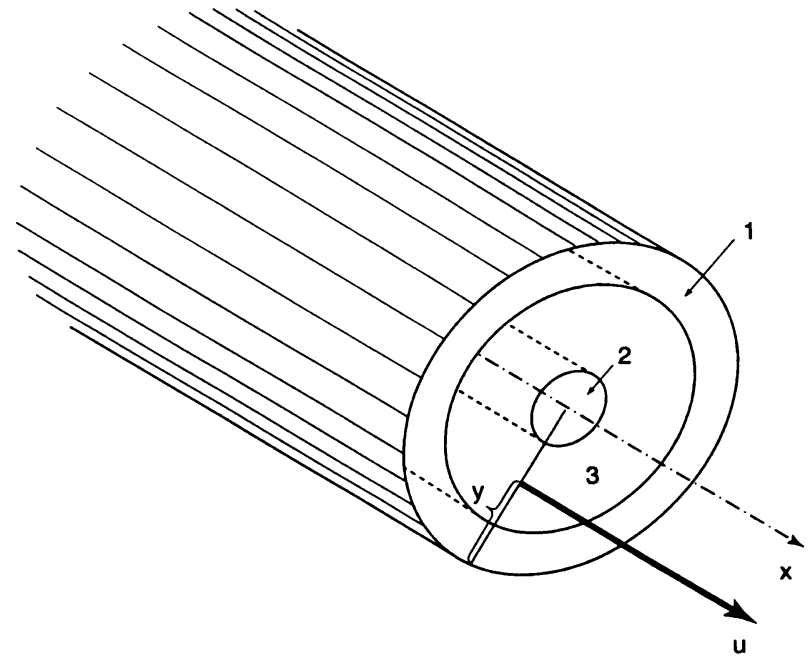

FIG. 1. Schematic view of the flow in a pipe: 1. Viscous sublayer; 2. Near-axis region; 3 . Intermediate region.

The velocity gradient $\partial_{y} u\left(\partial_{y} \equiv \frac{\partial}{\partial y}\right)$ in the intermediate region (3) of Fig. 1 depends on the following variables: the coordinate $y$, the shear stress $\tau$ at the wall, the pipe diameter $d$, the fluid's kinematic viscosity $\nu$, and its density $\rho$. We consider the velocity gradient $\partial_{y} u$ rather than $u$ itself because the values of $u$ depend on the flow in the viscous sublayer where the assumptions we shall make are not valid. Thus,

$$
\partial_{y} u=f(y, \tau, d, \nu, \rho) \text {. }
$$

Dimensional analysis gives

$$
\partial_{y} u=\frac{u_{*}}{y} \Phi(\eta, R e), \quad R e=\frac{\bar{u} d}{\nu}, \quad \eta=\frac{u_{*} y}{\nu},
$$

where $\Phi$ is a dimensionless function, or, equivalently,

$$
\partial_{\eta} \phi=\frac{1}{\eta} \Phi(\eta, R e), \quad \phi=\frac{u}{u_{*}} .
$$

Outside the viscous sublayer, $\eta$ is large, of the order of several tens and more; in the kind of turbulent flow that we consider, the Reynolds number $R e$ is also large, at least $10^{4}$. If one assumes that for such large values of $\eta$ and $R e$ the function $\Phi$ no longer varies with its arguments and can be replaced by its limiting value $\Phi(\infty, \infty)=\kappa^{-1}$ (this is an assumption of "complete similarity" in both arguments, see [1]), then Eq. (2.8) yields

$$
\partial_{\eta} \phi=\frac{1}{\kappa \eta},
$$

and then an integration yields the von Kármán-Prandtl "universal" logarithmic law [20, $23,28,30]$

$$
\phi=\frac{1}{\kappa} \ln \eta+B
$$


where $\kappa$ ("von Kármán's constant") and $B$ are assumed to be "universal", i.e., $R e$ independent constants. The assumption that $B$ is Reynolds-number-independent is an additional assumption. The resulting law is widely used to describe the mean velocity in the intermediate region; the values of $\kappa$ in the literature range between .36 and .44 and the values of $B$ between 5 and 6.3-an uncomfortably wide spread if one believes in the "universality" of (2.10).

However, there is no overwhelming reason to assume that the function $\Phi$ has a constant nonzero limit as its arguments tend to infinity, nor that the integration constant remains bounded as $R e$ tends to infinity. When either assumption fails, other conclusions must be reached. Rather than list alternative assumptions we present a model problem that exhibits in a simple manner what goes wrong as well as the cure.

Consider the equation

$$
\frac{d u}{d y}=\frac{1}{\ln (1 / \delta)} \frac{u}{y}
$$

for $y$ positive, where $u$ is subject to the boundary condition $u(\delta)=1$, and $\delta$ is a small positive parameter. One can view $\delta$ as a dimensionless viscosity, and thus $\delta^{-1}$ is analogous to a Reynolds number.

One could reason as follows: For $\delta$ small, $\frac{d u}{d y}$ is approximately zero, and thus $u$ is a constant, which can only be the constant 1 . We can derive the same result for small $y$ and $\delta$ by an assumption of complete similarity: Equation (2.11) is homogeneous in the dimensions of $u$ and $y$, and thus one can view both of these variables as dimensionless. By construction, $\delta$ must be dimensionless. The dimensionless relation between these variables takes the form

$$
u=\Phi(\delta, y)
$$

and if one makes an assumption of complete similarity, i.e., assumes that for $\delta, y$ small, $\Phi$ is constant, one finds again that $u$ is the constant 1 .

This conclusion is false. Equation (2.11) has the following solution that satisfies the boundary condition $u(\delta)=1$ :

$$
u(y)=\left(\frac{y}{\delta}\right)^{\frac{1}{\ln (1 / \delta)}} .
$$

Note that for any positive value of $\delta$ this solution is a power law and not a constant. We can obtain this solution for small $y$ and $\delta$ by assuming that the solution is a power of the variable $y$ while the form of its dependence on $\delta$ is unknown; this leads to $u=$ $A(\delta) y^{\alpha(\delta)}$. (This is an assumption of "incomplete similarity" in $y$ and no similarity in $\delta$.) A substitution into Eq. (2.11) yields $A(\delta), \alpha(\delta)$.

Further, consider the solution (2.13) and, for any nonzero value of $y$, its limit as $\delta \rightarrow 0$. Clearly,

$$
\left(\frac{y}{\delta}\right)^{\frac{1}{\ln (1 / \delta)}}=\exp \left(\frac{\ln (y / \delta)}{\ln (1 / \delta)}\right)=\exp \left(\frac{\ln (1 / \delta)+\ln (y)}{\ln (1 / \delta)}\right)
$$

and thus, as $\delta \rightarrow 0, u \rightarrow e$, i.e., the limit of (2.13) for $y>0$ is the constant $e$. As deduced from the false assumption of complete similarity, the limit of $u$ is a constant, but it is not the obvious constant 1 . Furthermore, for a finite value of $\delta$, however small, 
$u$ is not uniformly constant; it is not equal to $e$ either for $y<\delta$ or for $y$ large enough. The approximate equality $u \sim e$ holds, when $\delta$ is small but finite, only in an intermediate range where $y=O(1)$, and constitutes an example of "intermediate asymptotics" [1].

Now consider subjecting $\Phi$ in (2.8) to an analogous assumption, of incomplete similarity in $\eta$ and no similarity in $R e$ when both arguments are large [2]:

$$
\Phi(\eta, R e)=A(R e) \eta^{\alpha(R e)}
$$

Note a clear difference between complete and incomplete similarity. In the first case the experimental data should cluster in the $(\ln \eta, \phi)$-plane $\left(\phi=u / u_{*}, \eta=u_{*} y / \nu\right)$ on the single straight line of the logarithmic law; in the second case the experimental points would occupy an area in this plane. Both similarity assumptions are very specific. The possibility that $\Phi$ has no nonzero limit yet cannot be represented asymptotically since a power of $\eta$ has not been excluded. Both assumptions must be subjected to careful scrutiny. In the absence of reliable, high-Re numerical solutions of the Navier-Stokes equation and of an appropriate rigorous theory, this scrutiny must be based on comparison with experimental data.

We now specify the conditions under which (2.15) may hold and narrow down the choices of $A(R e)$ and $\alpha(R e)$ (see [4, 5, 6, 7, 9, 10]). Fully-developed turbulence is not a single, well-defined state with properties independent of $R e$; there may be such a single state in the limit of infinite Reynolds number, but experiment, even in the largest facilities, shows that fully-developed turbulence still exhibits a perceptible dependence on $R e$. Fully-developed turbulence has mean properties (for example, parameters such as $A$ and $\alpha$ in (2.15)) that vary with Reynolds numbers like $K_{0}+K_{1} \varepsilon$, where $K_{0}, K_{1}$ are constants and $\varepsilon$ is a small parameter that tends to zero as $R e$ tends to infinity, small enough so that its higher powers are negligible, yet not so small that its effects are imperceptible in situations of practical interest; the latter condition rules out choices such as $\varepsilon=(R e)^{-1}$. We expect $A(R e)$ and $\alpha(R e)$ in (2.15) to have the form

$$
A(R e)=A_{0}+A_{1} \varepsilon, \quad \alpha(R e)=\alpha_{0}+\alpha_{1} \varepsilon,
$$

where $A_{0}, A_{1}, \alpha_{0}, \alpha_{1}$ are universal constants; we have implicitly used a principle derived from the statistical theory of Sec. 4, according to which the average gradient of the velocity profile has a well-defined limit as the viscosity $\nu$ tends to zero $[5,6,10]$. This is the vanishing-viscosity principle. We expand $A(R e), \alpha(R e)$ in powers of $\varepsilon$ and keep the first two terms; the result is

$$
\Phi=\left(A_{0}+A_{1} \varepsilon\right) \eta^{\alpha_{0}+\alpha_{1} \varepsilon}
$$

Substitution of (2.17) into (2.15) yields

$$
\partial_{\ln \eta} \phi=\left(A_{0}+A_{1} \varepsilon\right) \eta^{\alpha_{0}+\alpha_{1} \varepsilon}=\left(A_{0}+A_{1} \varepsilon\right) e^{\left(\alpha_{0}+\alpha_{1} \varepsilon\right) \ln \eta} .
$$

For this expression to have a finite limit as $\nu$ tends to zero one needs $\alpha_{0}=0$, and $\varepsilon$ must tend to zero as $R e$ tends to infinity like $\left(\frac{1}{\ln R e}\right)$ or faster. The assumption of incomplete similarity, experiment, and the vanishing-viscosity principle show that the threshold value $\varepsilon=\frac{1}{\ln R e}$ is the proper choice. Use of this choice in Eq. (2.18) and an 
integration yield

$$
\phi=\frac{u}{u_{*}}=\left(C_{0} \ln R e+C_{1}\right) \eta^{\frac{\alpha_{1}}{\ln R e}}
$$

where the additional condition $\phi(0)=0$, motivated by the experimental data, has been used.

According to this derivation, the coefficients $C_{0}, C_{1}, \alpha_{1}$ are universal constants, the same in all experiments of sufficiently high quality performed in pipe flows at large Reynolds numbers [2, 3]. In [12] the proposed law for smooth walls (2.19) was compared with the data of Nikuradze [26] from Prandtl's institute in Göttingen. The comparison yielded the coefficients $\alpha_{0}=0, \alpha_{1}=3 / 2, C_{0}=\frac{1}{\sqrt{3}}, C_{1}=5 / 2$, with an error of less than $1 \%$. The result is

$$
\phi=\left(\frac{1}{\sqrt{3}} \ln R e+\frac{5}{2}\right) \eta^{3 /(2 \ln R e)} .
$$

We now wish to use the law (2.20) to understand what happens at larger Reynolds numbers and for a broader range of values of $\eta$ than were present in the experiments reported by Nikuradze. If this extrapolation agrees with experiment, we can conclude that the law has predictive powers and provides a faithful representation of the intermediate region. We have already stated that the limit that must exist for descriptions of the mean gradient in turbulent flow is the vanishing-viscosity limit, and thus one should be able to extrapolate the law (2.20) to ever smaller viscosities $\nu$. This is different from simply increasing the Reynolds number, since $\nu$ affects $\eta$ and $\bar{u}$ as well as $R e$. The decrease in the viscosity corresponds also to what is done in the experiments: If one stands at a fixed distance from the wall, in a specific pipe with a given pressure gradient, one is not free to vary $R e=\bar{u} d / \nu$ and $\eta=u_{*} y / \nu$ independently because the viscosity $\nu$ appears in both, and if $\nu$ is decreased by the experimenter, the two quantities will increase in a self-consistent way and $\bar{u}$ will vary as well. As one decreases the viscosity, one considers flows at ever larger $\eta$ at ever larger $R e$; the ratio $\frac{3 \ln \eta}{2 \ln R e}$ tends to $3 / 2$ because $\nu$ appears in the same way in both the numerator and the denominator. Consider the combination $3 \ln \eta /(2 \ln R e)$ in the form

$$
\frac{3 \ln \eta}{2 \ln R e}=\frac{3\left[\frac{u_{*} d}{\nu}+\ln \frac{y}{d}\right]}{2\left[\ln \frac{u_{*} d}{\nu}+\ln \frac{\bar{u}}{u_{*}}\right]} .
$$

According to [3], at small $\nu$, i.e., large $R e, \bar{u} / u_{*} \sim \ln R e$, so that the term $\ln \left(\bar{u} / u_{*}\right)$ in the denominator of the right-hand side of (2.21) is asymptotically small, of the order of $\ln \ln R e$, and can be neglected at large $R e$; because the viscosity $\nu$ is small, the first term $\ln \left(u_{*} d / \nu\right)$ in both the numerator and denominator of $(2.21)$ is dominant, as long as the ratio $y / d$ remains bounded from below, for example, by a predetermined fraction. Thus, away from a neighborhood of the wall, the ratio $3 \ln \eta /(2 \ln R e)$ is close to $3 / 2(y$ is obviously bounded by $d / 2$ ). Therefore

$$
1-\ln \eta / \ln R e
$$


is a small parameter as long as $y>\Delta$, where $\Delta$ is an appropriate fraction of $d$. The quantity $\exp (3 \ln \eta /(2 \ln R e))$ is approximately equal to

$$
\begin{aligned}
\exp \left[\frac{3}{2}-\frac{3}{2}\left(1-\frac{\ln \eta}{\ln R e}\right)\right] & \approx e^{3 / 2}\left[1-\frac{3}{2}\left(1-\frac{\ln \eta}{\ln R e}\right)\right] \\
& =e^{3 / 2}\left[\frac{3}{2} \frac{\ln \eta}{\ln R e}-\frac{1}{2}\right]
\end{aligned}
$$

According to $(2.20)$ we have also

$$
\eta \partial_{\eta} \phi=\partial_{\ln \eta} \phi=\left(\frac{\sqrt{3}}{2}+\frac{15}{4 \ln R e}\right) \exp \left(\frac{3 \ln \eta}{2 \ln R e}\right)
$$

and the approximation (2.22) can also be used in (2.23). Thus in the range of $y$ where $y>\Delta$, but $y<d / 2$, we find, up to terms that vanish as the viscosity tends to zero,

$$
\phi=e^{3 / 2}\left(\frac{\sqrt{3}}{2}+\frac{15}{4 \ln R e}\right) \ln \eta-\frac{e^{3 / 2}}{2 \sqrt{3}} \ln R e-\frac{5}{4} e^{3 / 2},
$$

and

$$
\partial_{\ln \eta} \phi=\frac{\sqrt{3}}{2} e^{3 / 2}
$$

Equation (2.25) is the asymptotic slope condition: As $\nu \rightarrow 0$ the slope of the power law tends to a finite limit, the limiting slope, which is the right-hand side of (2.25). The von Kármán-Prandtl law also subsumes an asymptotic slope condition, with a limiting slope $1 / \kappa$; the limiting slope in Eq. $(2.25), \frac{\sqrt{3}}{2} e^{3 / 2}=1 / .2776$, is approximately $\sqrt{e} \sim 1.65$ larger than a generally accepted value for $\kappa^{-1}$. One can view Eq. (2.24) as an asymptotic version of the classical logarithmic law, but with an additive term that diverges as the Reynolds number tends to infinity, and of course a different slope.

The family of curves $\phi=\phi(\eta)$ parametrized by $R e$ has an envelope whose equation tends to

$$
\phi=\frac{1}{\kappa} \ln \eta+\frac{5}{2} e
$$

with $\kappa=2 e / \sqrt{3}=.425 \ldots$, close to the values of $\kappa$ found in the literature. The corresponding value of $\frac{1}{\kappa}$ is exactly $\sqrt{e}$ times smaller than the value on the right-hand side of (2.25). It is clear that the logarithmic law usually found in the literature corresponds to this envelope; indeed, if one plots points that correspond to many values of $R e$ on a single graph (as is natural if one happens to believe the von Kármán-Prandtl law (2.10)), then one becomes aware of the envelope. The visual impact of the envelope is magnified by the fact that the small $y$ part of the graph, where the envelope touches the individual curves, is stretched out by the effect of $\nu$ on the values of $\ln \eta$. Also, measurements at very small values of $y$ where the difference between the power law and the envelope could also be noticeable are missing because of experimental difficulties very near the 


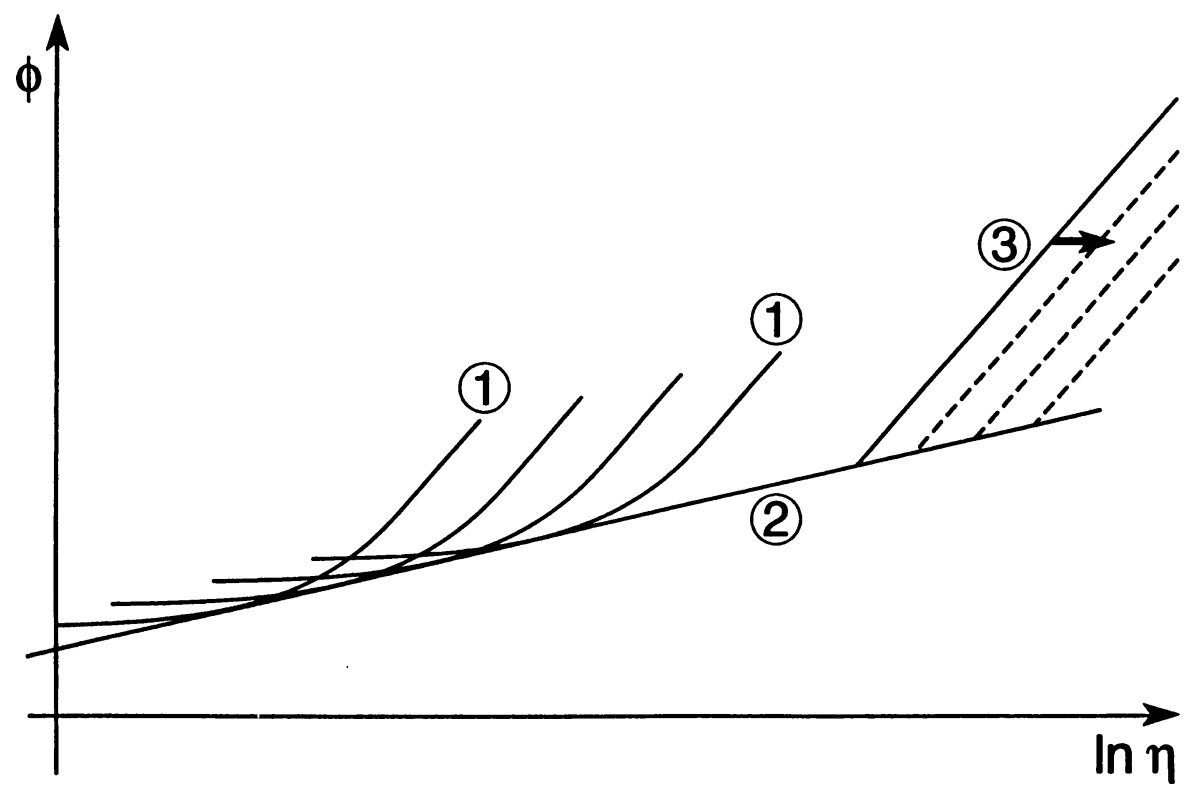

FIG. 2. Schematic of the power law curves, their envelope, and their asymptotic slope: 1 . The individual curves of the power law; 2 . The envelope of the family of power law curves (often mistaken for a logarithmic law of the wall); 3 . The asymptotic slope of the power law curves.

wall. Thus, if our proposed power law is valid, the conventional logarithmic law is an illusion that substitutes the envelope of the family of curves for the curves themselves. The discrepancy of $\sqrt{e}$ between the slope of the curves and the slope of the envelope is the signature of the power law, and helps to decide whether the power law is valid. The situation is summarized in Fig. 2, which shows schematically the individual curves of the power law, their envelope, and the asymptotic slope.

Historically, the understanding of the flow in the intermediate region of wall-bounded turbulence has been influenced by the "overlap" argument of Izakson, Millikan, and von Mises (IMM) (see, e.g., [25]). This argument in its original form contains an implicit assumption of complete similarity, and once freed from it yields yet again the asymptotic slope condition (2.25). For details, see [5, 7, 10].

Detailed comparisons of the power law and the von Kármán-Prandtl laws with experimental data are available in refs. [9, 10]. For completeness, we exhibit in Fig. 3 a set of experimental curves from the Princeton superpipe experiment [34]. Note its qualitative similarity to Fig. 2. In particular, despite a flaw discussed in detail in [10], these experiments do indeed exhibit a separate curve for each Reynolds number, and a well-defined angle between the curves and their envelope.

The applicability of our analysis of the intermediate region of pipe flow to the velocity profile in a zero-pressure-gradient boundary layer is discussed in [11]. 


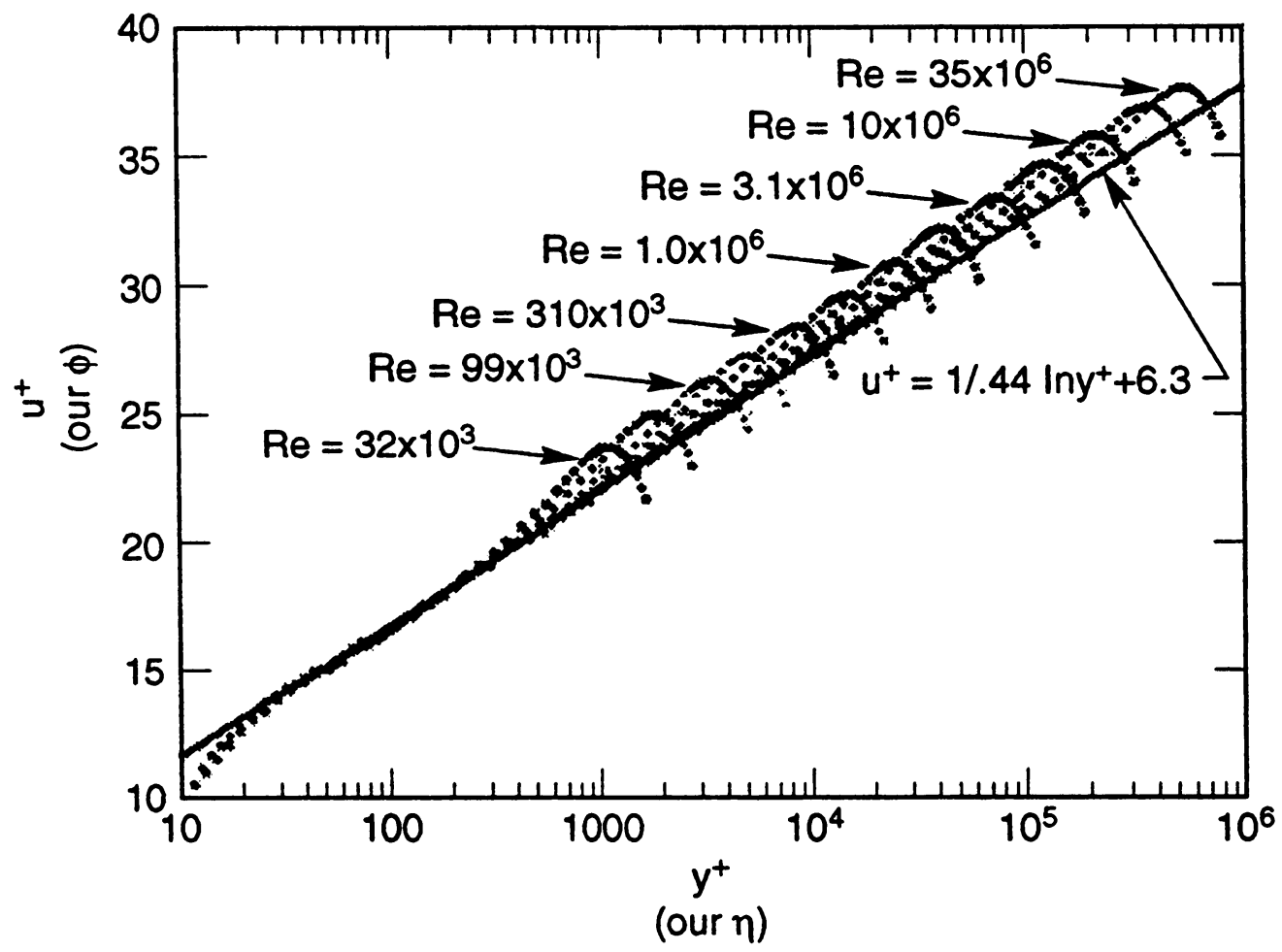

FIG. 3. The Princeton data [34] obtained in a high-pressure pipe confirm the splitting of the experimental data according to their Reynolds numbers and the rise of the curves above their envelope in the $(\ln \eta, \phi)$-plane. The solid line is the envelope; the curves turn at the center of the pipe. The splitting and form of the curves agree with the power law, and are incompatible with the von Kármán-Prandtl universal logarithmic law. (Reproduced with permission from [34]).

3. Local structure in turbulence. The analogy between the inertial range in the local structure of developed turbulence and the intermediate range in turbulent shear flow near a wall has been noted long ago (see, e.g., $[14,32]$, and it motivates the extension of the scaling analysis above to the case of local structure, where the experimental data are much poorer. In the problem of local structure, the quantities of interest are the moments of the relative velocity field, in particular, the second-order tensor with components

$$
D_{i j}=\left\langle\left(\Delta_{\mathbf{r}}\right)_{i}\left(\Delta_{\mathbf{r}}\right)_{j}\right\rangle
$$

where $\Delta_{\mathbf{r}}=\mathbf{u}(\mathbf{x}+\mathbf{r})-\mathbf{u}(\mathbf{x})$ is a velocity difference between $\mathbf{x}$ and $\mathbf{x}+\mathbf{r}$. In locally isotropic incompressible flow, all the components of this tensor are determined if one knows $D_{L L}=\left\langle\left[u_{L}(\mathbf{x}+\mathbf{r})-u_{L}(\mathbf{x})\right]^{2}\right\rangle$ where $u_{L}$ is the velocity component along the vector $\mathbf{r}$.

To derive an expression for $D_{L L}$ assume, following Kolmogorov, that for $r=|\mathbf{r}|$ small, it depends on $\langle\varepsilon\rangle$, the mean rate of energy dissipation per unit volume, $r$, the distance between the points at which the velocity is measured, a length scale $\Lambda$, for example the 
Taylor macroscale $\Lambda_{T}$, and the kinematic viscosity $\nu$ :

$$
D_{L L}(r)=f\left(\langle\varepsilon\rangle, r, \Lambda_{T}, \nu\right),
$$

where the function $f$ should be the same for all developed turbulent flows. If $r$ is large, other variables may appear, as a consequence of external forces or of boundary conditions. The most interesting and the most important argument in this list is the rate of energy dissipation $\varepsilon$.

Introduce the Kolmogorov scale $\Lambda_{K}$, which marks the lower bound of the "inertial" range of scales in which energy dissipation is negligible:

$$
\Lambda_{K}=\frac{\nu^{3 / 2}}{\langle\varepsilon\rangle^{1 / 4}} .
$$

Clearly, the velocity scale appropriate to the inertial range is

$$
u=\left(\langle\varepsilon\rangle \Lambda_{T}\right)^{1 / 3},
$$

and this yields a Reynolds number

$$
R e=\frac{\left(\langle\varepsilon\rangle \Lambda_{T}\right)^{1 / 3} \Lambda_{T}}{\nu}=\frac{\langle\varepsilon\rangle^{1 / 3} \Lambda_{T}^{4 / 3}}{\nu}=\left(\frac{\Lambda_{T}}{\Lambda_{K}}\right)^{4 / 3} .
$$

The inertial range of scales is intermediate between the scales on which the fluid is stirred and the scales where viscosity dissipates energy, and is the analog of the intermediate region in wall-bounded flow. In this range the scaling law that corresponds to $(2.15)$ is

$$
D_{L L}=(\langle\varepsilon\rangle r)^{\frac{2}{3}} \Phi\left(\frac{r}{\Lambda_{K}}, R e\right),
$$

where as before, the function $\Phi$ is a dimensionless function of its arguments, which have been chosen so that, under the circumstances of interest here, they are both large.

If one now subjects (3.6) to an assumption of complete similarity in both its arguments, one obtains the classical Kolmogorov 2/3 law [21],

$$
D_{L L}=A_{0}(\langle\varepsilon\rangle r)^{\frac{2}{3}},
$$

from which the Kolmogorov-Obukhov " $5 / 3$ " spectrum [27] can be obtained via Fourier transform. If one makes the assumption of incomplete similarity in $r / \Lambda_{K}$ and no similarity in $R e$, as in the case of wall-bounded flow, the result is

$$
\frac{D_{L L}(r)}{(\langle\varepsilon\rangle r)^{2 / 3}}=C(R e)\left(\frac{r}{\Lambda_{K}}\right)^{\alpha(R e)},
$$

where $C, \alpha$ are functions of $R e$ only. As before, expand $C$ and $\alpha$ in powers of $\frac{1}{\ln R e}$ and keep the two leading terms; this yields

$$
D_{L L}=(\langle\varepsilon\rangle r)^{2 / 3}\left(C_{0}+\frac{C_{1}}{\ln R e}\right)\left(\frac{r}{\Lambda_{K}}\right)^{\alpha_{1} / \ln R e}
$$

( $\alpha_{0}$ has been set equal to zero so that $D_{L L}$ has a finite limit as $\nu \rightarrow 0$ ).

In real measurements for finite but accessibly large $R e, \alpha_{1} / \ln R e$ is small in comparison with $2 / 3$, and the deviation in the power of $r$ in (3.9) could be unnoticeable. On the other hand, the variations in the "Kolmogorov constant" have been repeatedly noticed 
(see $[25,29,31])$. Complete similarity is possible only if $A_{0} \neq 0$, when one has a welldefined turbulent state with a 2/3 law in the limit of vanishing viscosity, and finite $R e$ effects can be obtained by expansion about that limiting state. In the limit of vanishing viscosity there are no corrections to the "K41" scaling if Eq. (3.9) holds; this conclusion was reached in [15] by the statistical mechanics argument summarized in Sec. 4 below.

Kolmogorov [21] proposed similarity relations also for the higher-order structure functions:

$$
D_{L L \ldots L}(r)=\left\langle\left[u_{L}(\mathbf{x}+\mathbf{r})-u_{L}(\mathbf{x})\right]^{p}\right\rangle,
$$

where $L L \ldots L$ denotes $L$ repeated $p$ times; the scaling gives $D_{L L \ldots L}=C_{p}(\langle\varepsilon\rangle r)^{p / 3}$. Experiments in a small wind-tunnel, by Benzi et al. [13], show some self-similarity in these higher-order functions, obviously incomplete, so that $D_{L L \ldots L}$ is proportional to $r^{\zeta_{p}}$, with exponents $\zeta_{p}$ always smaller then $p / 3$ for $p \geq 3$, so that $\zeta_{4}=1.28$ instead of $1.33, \zeta_{5}=1.53$ instead of $1.67, \zeta_{6}=1.77$ instead of $2.00, \zeta_{7}=2.01$ instead of 2.33 , and $\zeta_{8}=2.23$ instead of 2.67. It is tempting to try for an explanation of the same kind as for $p=2$ :

$$
D_{L L \ldots L}=\left(C_{p}^{0}+\frac{C_{p}^{1}}{\ln R e}\right)\left(\langle\varepsilon r\rangle^{p / 3}\left(r / \Lambda_{K}\right)^{\alpha_{p} / \ln R e}\right)
$$

in other words, to assume that at $R e=\infty$ the classic "K41" theory is valid, but the experiments are performed at Reynolds numbers too small to reveal the approach to complete similarity. If this explanation were correct, the coefficients $\alpha_{p}$ would be negative starting with $p=4$, where there would be a reversal in the effect of the Kolmogorov scale (or whatever scale is used to scale the first argument in $\Phi$ ).

As is well known, for $p=3$ the Kolmogorov scaling is valid with no corrections. For $p>3$ one must proceed with caution. We would like to present a simple argument that casts doubt on the good behavior of the structure functions for $p>3$ in the vanishingviscosity limit. As $R e \rightarrow \infty$ the intense vorticity and the large velocities in the fluid become concentrated in an ever-smaller volume [15]. This is what we call "intermittency". If $V_{0}$ is the fraction of the volume of a unit mass of fluid where the kinetic energy $\approx u^{2}$ is large, then $u \approx \frac{1}{\sqrt{V_{0}}}$; one can see that fourth moments such as $\left\langle u^{4}\right\rangle$ diverge as $V_{0} \rightarrow 0$. This casts a strong doubt on the good behavior of the fourth-order structure functions as the viscosity tends to zero; in the absence of such good behavior our expansions in powers of $\frac{1}{\ln R e}$ cannot be justified and the explanation of the experimental data must proceed along different lines. Note that $p=3$ is the power where the sign of the power of $\frac{r}{\Lambda_{K}}$ in an expansion in powers of $\frac{1}{\ln R e}$ would change.

The analysis just given of the second-order structure function contradicts the conclusions of Benzi et al. [13], according to whom the asymptotic exponent in (3.9) is independent of $R e$ and different from $2 / 3$. We wish to point out however that, as we understand the discussion in [13], the exponent was found to be different from $2 / 3$ only once it was assumed that it was not dependent on $R e$; to the contrary, even a cursory view of Fig. 3 in ref. [13] shows a marked dependence on Re. We are looking forward to an opportunity to re-examine these data in the light of our hypotheses. 
There is a key difference between a derivation of the Kolmogorov-Obukhov exponent from an assumption of complete similarity and its derivation as the vanishing-viscosity limit of an expression derived from an assumption of incomplete similarity. Complete similarity typically holds in statistical problems that are well-described by mean-field theories, while incomplete similarity typically applies to problems where fluctuations are significant. This remark is consistent with our conclusion, presented in $[7,10]$, that the Kolmogorov scaling already allows for intermittency, and that its application to higherorder structure functions is limited by this very intermittency.

4. A near-equilibrium theory of turbulence. At large Reynolds numbers $R e$ the solutions of the Navier-Stokes equations are chaotic, and the slightest perturbation alters them greatly. The proper object of a theory of turbulence is the study of ensembles of solutions, i.e., of collections of solutions with probability distributions that describe the frequency of their occurrence. We now outline a near-equilibrium theory of ensembles of flows on those small scales where the scaling theory of the previous section applies. This theory justifies the use of vanishing-viscosity asymptotics for appropriate moments of the velocity field and of its derivatives and supports the conclusions of the previous section regarding the behavior of the higher-order moments and structure functions. It is equivalent to earlier near-equilibrium theories [15], but the specific approach and the presentation are new; fuller detail can be found in [8].

We describe turbulence in terms of a suitable statistical equilibrium. In statistical mechanics, statistical equilibrium is what one finds in an isolated system if one waits long enough. One way of characterizing this equilibrium is by assuming that all states of the system compatible with the system's given energy can occur with equal probabilities; this is the "microcanonical ensemble". In turbulence the appropriate energy is the kinetic energy of the flow. An equivalent characterization is in terms of the "canonical ensemble", in which the probability of a state is proportional to $\exp (-\beta H)$, where $H$ is the energy of the state and $\beta$ is a parameter. In the canonical ensemble the energy is not fixed, and one can view the ensemble as describing a portion of an isolated system at equilibrium as it interacts with the rest of the system. The two ensembles are equivalent in the sense that, with a proper choice of the parameter $\beta$ and in a system with enough degrees of freedom, averages calculated in either ensemble are close to each other.

The parameter $\beta$ is generally called the "inverse temperature" of the system. In many physical systems $T=1 / \beta$ is indeed proportional to what one intuitively perceives to be the temperature, as it can be gauged by touching a system with one's finger. However, the parameter $\beta$ can be viewed more abstractly, as the parameter that makes the two ensembles equivalent; in incompressible turbulence, in which there is no interaction between the macroscopic flow and the microscopic motion of the molecules of the fluid, the $\beta$ that one obtains cannot be gauged by the sense of touch. In a given system, $\beta$ is a function of the energy $E$ and of whatever other variables are needed to describe the system. Note that in other realms of physics, for example, in the kinetic theory of gases, one is well used to relating temperature to a suitable kinetic energy.

Turbulence as a whole is generally not an equilibrium phenomenon: For example, if one stirs a box full of fluid and then isolates the resulting flow, the outcome after a long 
time is not turbulence in a statistical equilibrium but a state of rest; an isolated turbulent flow is one without outside forces or an imposed shear to keep it flowing. However, on the small scales in which we are interested, the relevant question is whether the motion has enough time to settle to an approximation of a statistical equilibrium in which one can assume that all the states with a given kinetic energy are equally likely to appear. The small scales have enough time when their characteristic time (length/velocity) is short enough compared to the characteristic time of the large-scale motion. An inspection of the Kolmogorov scaling given in the previous section shows that the characteristic time of an eddy of size $r$ is proportional to $r^{2 / 3}$, and small enough eddies (i.e., vortices) do have enough time to settle down to an equilibrium distribution. The task at hand is to construct the statistical equilibrium appropriate for turbulence, in particular, specify its states. The question of how then to perturb it so as to take into account the irreversible aspects of turbulence has been treated elsewhere $[16,17]$ and will not concern us here. Note that in most of the turbulence literature one speaks of the small scales reaching "equilibrium" when the energy distribution among them approximates the KolmogorovObukhov form; here, for the moment, we mean by "equilibrium" a statistical equilibrium, in which all states have equal probability; we shall shortly claim that these two meanings are in fact identical. This is of course possible only if at the statistical equilibrium there are more states with much of the energy in the larger scales than states with much of the energy in the smaller scales.

To agree with observation, a hydrodynamical statistical equilibrium must have a finite energy density in physical space. To construct an equilibrium with this property, we start as in the construction employed in ref. [24] for vorticity fields; for simplicity we describe it in two space dimensions. Consider a unit box $0 \leq x \leq 1,0 \leq y \leq 1$, with periodic boundary conditions. Let $\mathbf{u}=(u, v)$ be the velocity field and $\psi$ a stream function; divide the box into $N^{2}$ squares of side $h, N h=1$; in each square define a value of a discrete stream function $\psi_{i j}$, where $i, j$ describe the location of the square; then define a discrete velocity field $\mathbf{u}_{i j}$ by one-sided difference quotients of $\psi$, so that the velocity is divergencefree. (In three dimensions there is one more index and the stream function is replaced by a vector potential.) The parameter $h$ is an artificial cut-off, and we now present a procedure for letting this cut-off tend to zero while producing sensible fluid mechanics in the limit.

Replace the energy $E=\int_{\text {square }}|\mathbf{u}|^{2} d x d y$ by its discrete counterpart $E_{h}=\sum\left|\mathbf{u}_{i j}^{2}\right| h^{2}$. For a fixed value of $h$, pick a value of $E_{h}$, and, as a first step, assume that the values of $\mathbf{u}$ are equidistributed among all states with $E_{h}=$ constant, i.e., use a microcanonical ensemble. One can check that on the average, each one of the boxes has the same energy $\left|\mathbf{u}_{i j}\right|^{2} h^{2}$.

One may think that if one lets $h \rightarrow 0$ while keeping $E_{h}$ constant, the limit is an ensemble with a finite energy per unit volume; this is not so. The sequence of ensembles one obtains as $h \rightarrow 0$ has no reasonable limit: As $h \rightarrow 0$ the number of degrees of freedom tends to infinity, and there is no sensible way to divide a finite energy equally among an infinite number of degrees of freedom; indeed, if the energy per degree of freedom is zero the limiting ensemble has zero energy and no motion, and if the energy per degree of freedom is positive the limiting ensemble has an infinite energy (for a more thorough 
mathematical discussion, see $[6,8])$. One can also see that the limit of these microcanonical ensembles is meaningless by considering the corresponding canonical ensembles: One can check that as $h \rightarrow 0$ the parameter $\beta$ in the sequence of canonical ensembles tends to infinity; one can show that the only ensembles with infinite $\beta$ have either no energy or an infinite energy. To find a way out of this dilemma one must modify these ensembles as $h \rightarrow 0$ so as to ensure that the limit exists. We do so by looking at what happens to the parameter $\beta$ and keeping it bounded; furthermore, we do so on the computer. This is the key point: To obtain a sensible continuum limit, we keep $\beta$ bounded by keeping the energy from becoming equally distributed among the degrees of freedom, and this produces an average energy distribution among scales that agrees with the KolmogorovObukhov law and produces the Kolmogorov scaling of the low-order structure functions. One can also show (see [8]) that this very same procedure is needed to produce ensembles whose members, the individual velocity fields, do not violate what is known about the solutions of the Navier-Stokes or Euler equations.

To proceed, we have to be able to calculate $\beta$ given $h$ and $E_{h}$. Averages with respect to microcanonical ensembles can be calculated numerically by an algorithm known as "microcanonical sampling" [18]: Introduce an additional variable, a "demon", which interacts with all the degrees of freedom in some random order. In each interaction, the demon either absorbs an energy packet of some predetermined magnitude $s, s \ll E_{h}$, or gives away an energy packet of the same size. If the demon takes in an energy $s$, it reduces the energy in the velocity field by modifying $\psi_{i j}$ so that the integral $\int|\mathbf{u}|^{2} d x d y$ over the unit square is reduced by $s$; the effect of this reduction modifies the values of $\mathbf{u}$ in the neighborhood of the point $(i, j)$. If the demon gives out energy, it modifies $\psi$ so as to increase the energy integrai. The demon is constrained so that it cannot give out energy unless it had acquired energy in its previous history; no "loans" are allowed. The sequence of states wrought by the demon's actions ranges over even-handedly the configurations of the system. If one wishes to conserve an additional quantity, as we shall below, one can do so by allowing the demon to exchange doses of the conserved quantity as it wanders along, subject to the condition that it never give out what it does not have.

The parameter $\beta$ in the equivalent canonical ensemble can be determined in the course of calculating averages: As the demon interacts with the ensemble it typically has some energy stored away; the system consisting of the physical system plus the demon is isolated, and by the equivalence of the canonical and microcanonical ensembles, the probability of an energy $E_{d}$ being stored by the demon is canonical, i.e., proportional to $\exp \left(-\beta E_{d}\right)$; this observation allows one to estimate $\beta$ after the demon has had a sufficient number of interactions. In addition to its dynamical role in moving the system from state to state for the purpose of calculating averages, the demon reveals the value of $\beta$; if $h$ and $E_{h}$ are given, there is a well-defined numerical procedure for finding the corresponding $\beta$.

Rather than keep $\beta$ merely bounded, we keep it constant. To do this, one needs a variable that can be altered and whose variation controls $\beta$. Experience and mathematics show that one can use as control variable the integral $I=\int|\xi| d x d y$, where $\xi$ is the vorticity calculated by finite differences and the integral is approximated by the appropriate 
sum. Thus the plan is to determine an $I$ for each $h$ so as to $\operatorname{keep} \beta$ at a fixed value $\beta_{\text {goal }}$ common to all the $h$. For simplicity and without loss of generality we set $E_{h}=1$.

For a given $h$, pick a starting guess for $I$, say $I_{0}$, and then produce a sequence of better values $I_{n}, n=1,2, \ldots$ by the formula

$$
I_{n+1}=I_{n}-K\left(\beta-\beta_{\text {goal }}\right),
$$

where $\beta$ without a subscript is the latest estimate of $\beta$ available from the demon and $K$ is a numerical parameter chosen so as to ensure that the $I_{n}$ converge to a limit. Before calculating a new value $I_{n+1}$ of $I$ the demon must be allowed at least one energy exchange with the ensemble, during which the variable $I$ is maintained at its last value $I_{n}$. Once $\beta$ reaches the desired value $\beta_{\text {goal }}$ the quantity $I$ remains constant. The resulting ensemble gives nonzero, equal probabilities to all states compatible with both the given value $E_{h}$ and the calculated value of $I$; when both constraints are satisfied, the energy per degree of freedom is no longer the same for all the degrees of freedom.

The changes in $I$ needed to keep $\beta$ fixed as $h$ is changing are displayed in Fig. 4 for several values of $\beta=\beta_{\text {goal }}$. The statistical error throughout is of the order of $2 \%$. The values of $I$ needed to keep $\beta$ fixed increase with $N=1 / h$. As shown in $[8,16]$, for small enough $h$ the curves are independent of the value of $\beta$, and this fact is reflected in the confluence of the several curves in Fig. 4. Note that $I$ is calculated on the grid by taking differences of the values of the velocity $u$ at points separated by $h$, which by the Kolmogorov-Obukhov scaling should be proportional to $h^{1 / 3}$; then one divides by $h$, and takes an average; one expects $I$ to grow with $N$ like $N^{2 / 3}$. In Fig. 5 we plot the logarithm of $I$ vs. the logarithm of $N$; the relation is well approximated by a straight line whose slope is .65 with an error of \pm .05 . Within the limitations of the Monte-Carlo sampling, the Kolmogorov-Obukhov scaling is seen to be applicable in this equilibrium model. Since the Kolmogorov-Obukhov scaling applies to the low-order structure functions in a flow with a finite but small viscosity, Fig. 5 shows that low-order moments structure functions have a limit as the viscosity tends to zero. One can perform a similar analysis of the small-scale structure of flow near a wall and conclude that the first-order moments of the derivatives of the velocity field near walls have well-behaved limits, a fact used above in the discussion of the scaling of the wall-region in the pipe. Figure 4 defines the limiting process in which $h \rightarrow 0$ with a limit that provides a meaningful equilibrium ensemble for the small scales of the flow.

The fact that the construction above is numerical enhances its value rather than detracts from it, as we expect to use similar constructions in the numerical modeling of turbulence. An elegant argument, suggested by the work of Kailasnath et al. [19] and presented in detail in [8], shows that the third-order structure function is calculated in the equilibrium theory exactly; of greater interest here is what happens to moments of the velocity field of order four and more. We have argued in the preceding section that the vanishing viscosity limit is not well-behaved for these higher moments, and thus the good behavior of the structure functions is unlikely and the expansion in powers of $1 / \ln R e$ is invalid. In the equilibrium theory the fourth-order moments fail to converge 


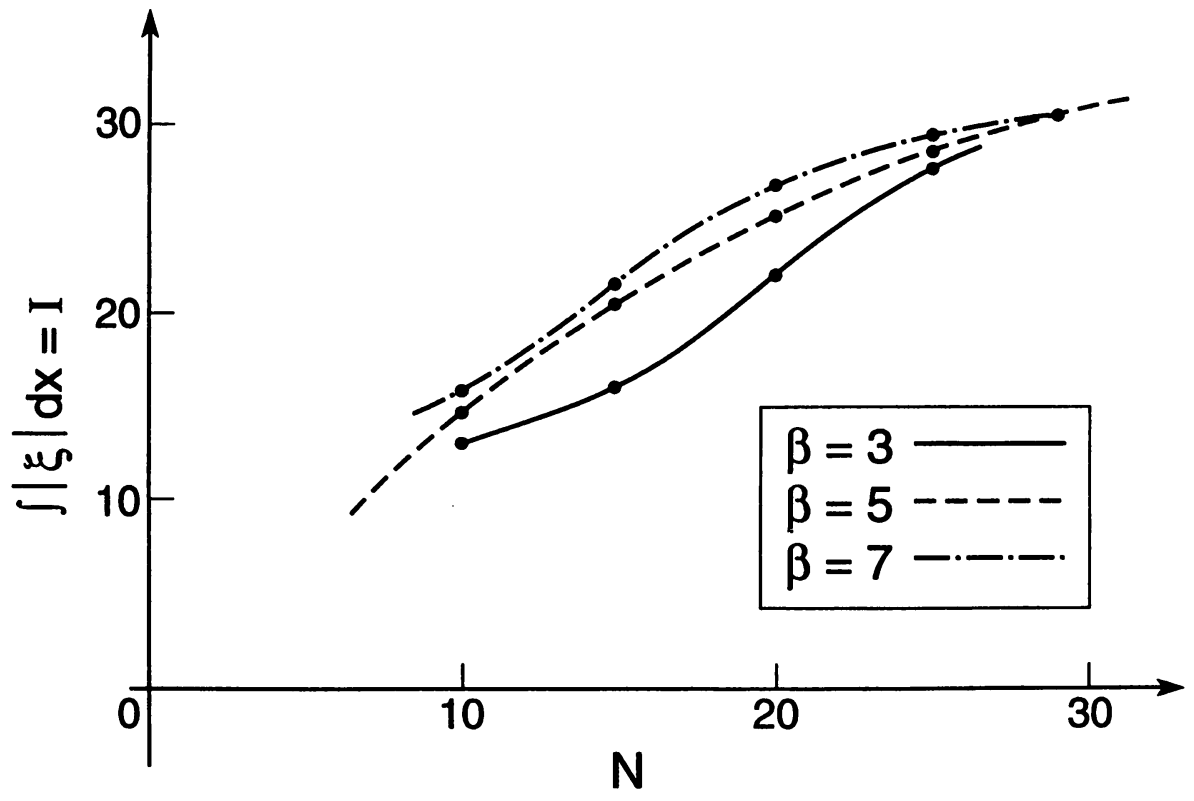

FIG. 4. The variation of $I=\int|\xi| d x$ with $N$ needed to keep $\beta$ fixed, for several values of $\beta$

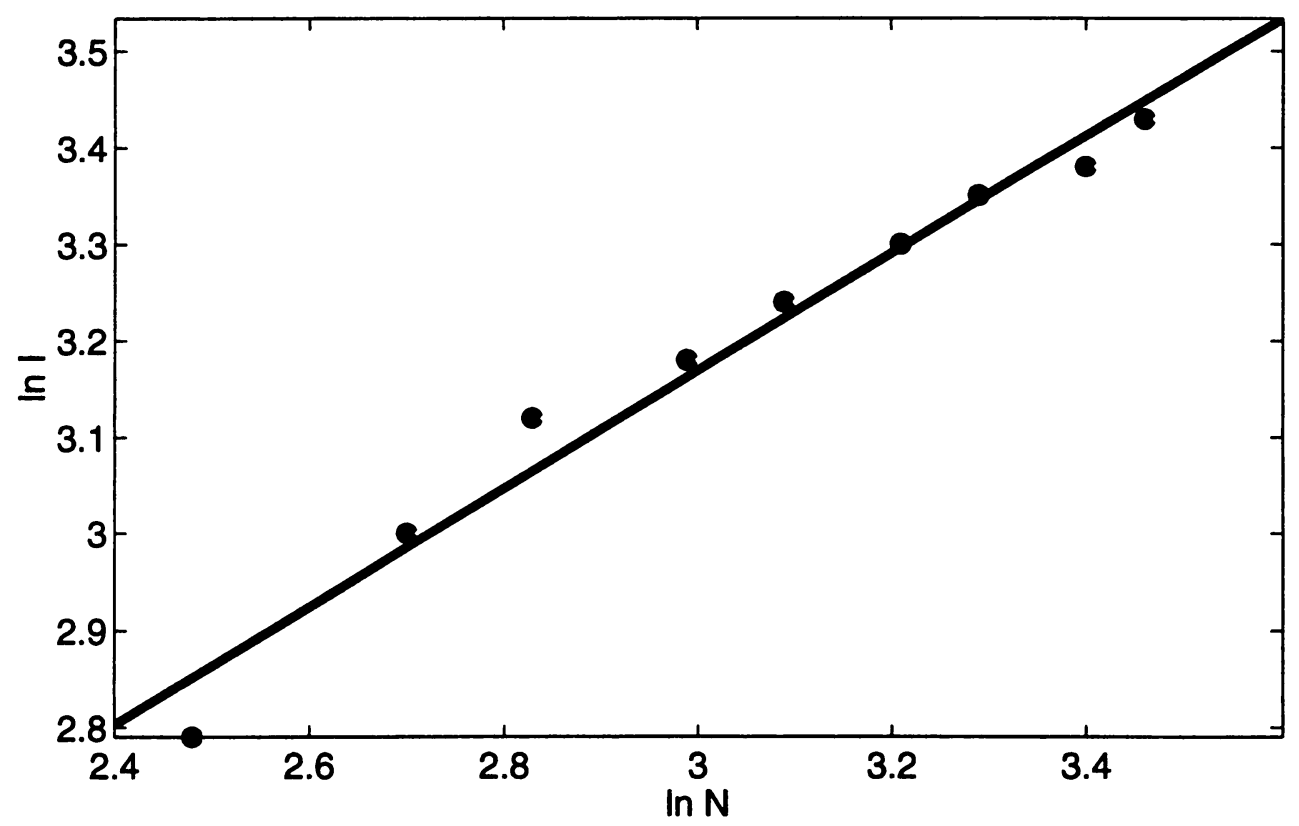

FIG. 5. The variation of $\ln I$ vs. $\ln N$ for $\beta=5$ exhibits a linear regime and a slope given by the Kolmogorov-Obukhov scaling. 


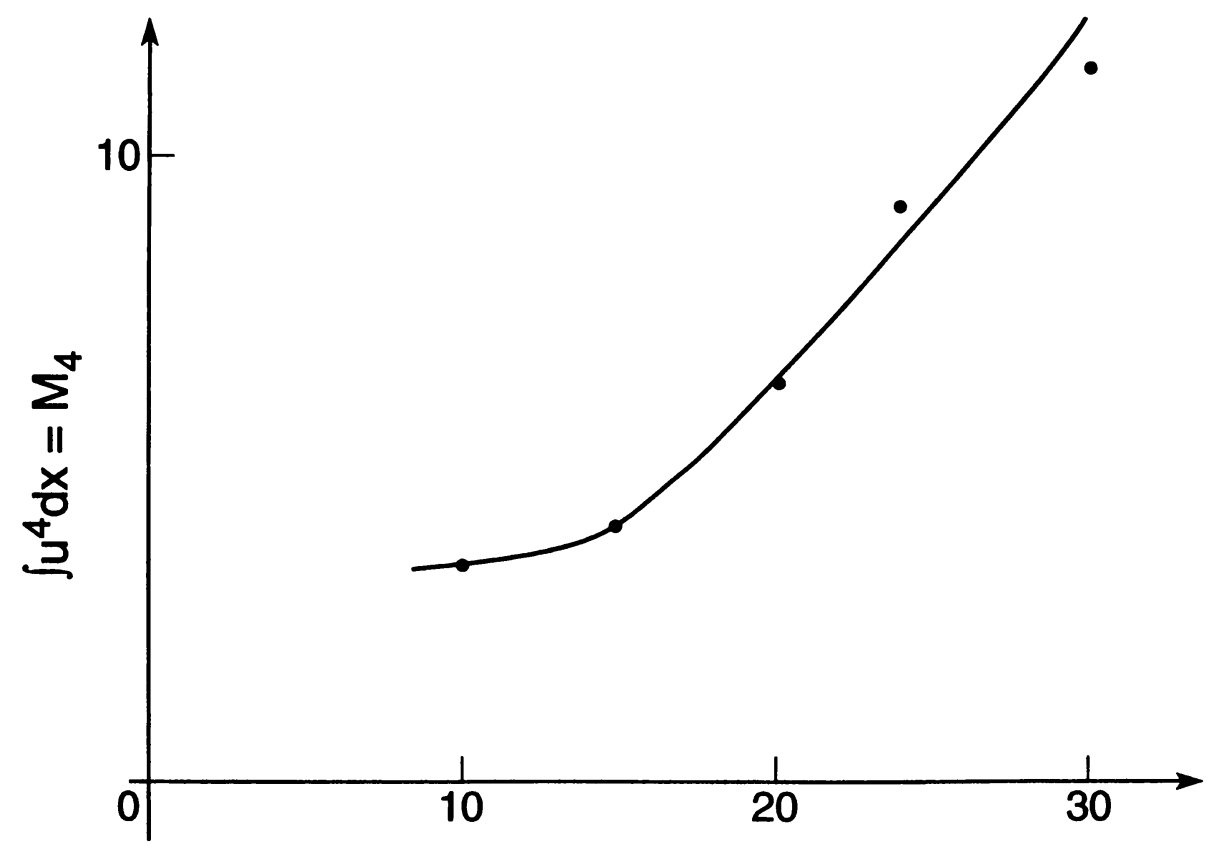

FIG. 6. The growth of the fourth moment of the velocity field as $N$ increases

to a finite limit as $N=1 / h \rightarrow \infty$. In Fig. 6 we display the fourth-order moment $\int|\mathbf{u}|^{4} d x d y$ as a function of $N$ at the parameter value $\beta=5$. Higher-order moments diverge even faster. The divergence of the higher moments corresponds to the formation of concentrated vortical structures, like the ones explicitly constructed in [16]. We have thus produced Kolmogorov scaling for the low-order moments in a system that is highly intermittent in the sense that the vorticity is concentrated on a small fraction of the available volume. The results of the equilibrium theory are therefore consistent with the scaling analysis of the previous section, according to which the Kolmogorov scaling of the second-order structure function is exact in the limit of vanishing viscosity not despite intermittency but because of intermittency, while its failure for the higher-order moments can be ascribed to the absence of a well-behaved vanishing-viscosity limit, as a result of which the expansion in the inverse powers of $\ln R e$ is not legitimate.

Note the small number of assumptions made in the equilibrium theory; all that was assumed was that the fluid was near statistical equilibrium on the small scales, the fluid was incompressible, the energy density in physical space was finite, and a probability measure on the ensemble of flows was well-defined. The Navier-Stokes equations did not enter the argument in the present paper (but see ref. [8]).

Finally, it is worth noting that an analysis of simplified near-equilibrium vortex models $[22,33]$ has provided an example where an expansion in powers of a parameter analogous to $1 / \ln R e$ can be fully justified without recourse to experimental data. 
5. Conclusions. We have reached the following conclusions:

(1) The von Kármán-Prandtl law of the wall must be jettisoned, and replaced by a power law with a Reynolds-number dependent coefficient and exponent, as suggested by an assumption of incomplete similarity.

(2) The Kolmogorov-Obukhov scaling of low-order structure functions in the local structure of turbulence admits only viscosity-dependent corrections, which vanish as the Reynolds number tends to infinity. There are no "intermittency corrections" to this scaling in the limit of vanishing viscosity. The Kolmogorov scaling of the higher-order structure functions fails because of intermittency.

(3) These conclusions are consistent with and support the near-equilibrium theory of turbulence.

Acknowledgment. Prof. Barenblatt and I would like to thank the following persons for helpful discussions and comments and/or for permission to use their data: Prof. N. Goldenfeld, Prof. O. Hald, Dr. M. Hites, Prof. F. Hussain, Dr. A. Kast, Dr. R. Kupferman, Prof. H. Nagib, Prof. C. Wark, and Dr. M. Zagarola.

\section{REFERENCES}

[1] G. I. Barenblatt, Similarity, Self-Similarity and Intermediate Asymptotics, Consultants Bureau, NY, 1979; Scaling, Self-Similarity, and Intermediate Asymptotics, Cambridge Texts in Applied Mathematics, vol. 14, Cambridge University Press, Cambridge, 1996

[2] G. I. Barenblatt, On the scaling laws (incomplete self-similarity with respect to Reynolds number) in the developed turbulent flow in pipes, C. R. Acad. Sci. Paris, series II, 313, 3.9-3.12 (1991)

[3] G. I. Barenblatt, Scaling laws for fully developed turbulent shear flows. Part 1: Basic hypotheses and analysis, J. Fluid Mech. 248, 513-520 (1993)

[4] G. I. Barenblatt and A. J. Chorin, Small viscosity asymptotics for the inertial range of local structure and for the wall region of wall-bounded turbulence, Proc. Nat. Acad. Sciences USA 93, 6749-6752 (1996)

[5] G. I. Barenblatt and A. J. Chorin, Scaling laws and vanishing-viscosity limits for wall-bounded shear flows and for local structure in developed turbulence, Comm. Pure Appl. Math. 50, 381-398 (1997)

[6] G. I. Barenblatt and A. J. Chorin, Scaling laws and vanishing viscosity limits in turbulence theory, Proc. Venice Conf. in honor of P. Lax and L. Nirenberg, Proc. Sympos. Appl. Math., Vol. 54, Amer. Math. Soc., Providence, RI, 1998, pp. 1-25

[7] G. I. Barenblatt and A. J. Chorin, New perspectives in turbulence: Scaling laws, asymptotics and intermittency, SIAM Rev. 40, 265-291 (1998)

[8] G. I. Barenblatt and A. J. Chorin, A near-equilibrium statistical theory of turbulence with applications, in preparation, 1998

[9] G. I. Barenblatt, A. J. Chorin, and V. M. Prostokishin, Scaling laws in fully developed turbulent pipe flow: Discussion of experimental data, Proc. Nat. Acad. Sciences USA 94a, 773-776 (1997)

[10] G. I. Barenblatt, A. J. Chorin, and V. M. Prostokishin, Scaling laws in fully developed turbulent pipe flow, Appl. Mech. Rev. 50, 413-429 (1997)

[11] G. I. Barenblatt, A. J. Chorin, O. H. Hald, and V. M. Prostokishin, Structure of the zero-pressuregradient turbulent boundary layer, Proc. Nat. Acad. Sciences USA 94, 7817-7819 (1997)

[12] G. I. Barenblatt and V. M. Prostokishin, Scaling laws for fully developed turbulent shear flows. Part 2. Processing of experimental data, J. Fluid Mech. 248, 521-529 (1993)

[13] R. Benzi, C. Ciliberto, C. Baudet, and G. Ruiz Chavarria, On the scaling of three dimensional homogeneous and isotropic turbulence, Physica D 80, 385-398 (1995)

[14] A. J. Chorin, Theories of turbulence, in Berkeley Turbulence Seminar, edited by P. Bernard and T. Ratiu, Springer, NY, 1977

[15] A. J. Chorin, Vorticity and Turbulence, Springer, NY, 1994

[16] A. J. Chorin, Turbulence as a near-equilibrium process, Lectures in Appl. Math. 31, 235-248 (1996) 
[17] A. J. Chorin, Turbulence cascades across equilibrium spectra, Phys. Rev. E 54, 2615-2619 (1996)

[18] H. Gould and J. Tobochnik, An Introduction to Computer Simulation Methods, Applications to Physical Systems, part 2, Addison-Wesley, Reading, MA, 1988

[19] P. Kailasnath, A. Migdal, K. Sreenivasan, V. Yakhot, and L. Zubair, The 5/4 Kolmogorov law and odd moments of the velocity difference in turbulence, unpublished, 1992

[20] Th. von Kármán, Mechanische Aehnlichkeit und Turbulenz, Nach. Ges. Wiss. Goettingen MathPhys. Klasse, 1932, pp. 58-76

[21] A. N. Kolmogorov, Local structure of turbulence in incompressible fluid at a very high Reynolds number, Dokl. Acad. Sci. USSR 30, 299-302 (1941)

[22] R. Kupferman and A. J. Chorin, Numerical study of the Kosterlitz-Thouless phase transition, submitted for publication, 1997

[23] L. D. Landau and E. M. Lifshitz, Fluid Mechanics, Pergamon Press, New York, 1959

[24] J. Miller, Statistical mechanics of the Euler equation in two dimensions, Phys. Rev. Lett. 65, 2137-2141 (1990)

[25] A. S. Monin and A. M. Yaglom, Statistical Fluid Mechanics, Vol. 1, MIT Press, Boston, 1971

[26] J. Nikuradze, Gesetzmaessigkeiten der turbulenten Stroemung in glatten Rohren, VDI Forschungheft, No. 3.8, 1932

[27] A. M. Obukhov, Spectral energy distribution in turbulent flow, Dokl. Akad. Nauk USSR 1, 22-24 (1941)

[28] L. Prandtl, Zur turbulenten Stroemung in Rohren und laengs Platten, Ergeb. Aerodyn. Versuch., Series 4, Goettingen, 1932

[29] A. Praskovsky and S. Oncley, Measurements of the Kolmogorov constant and intermittency exponents at very high Reynolds numbers, Phys. Fluids A 7, 2778-2784 (1994)

[30] H. Schlichting, Boundary Layer Theory, McGraw-Hill, NY, second edition, 1968

[31] K. R. Sreenivasan, On the universality of the Kolmogorov constant, Phys. Fluids A 7, 2778-2784 (1995)

[32] H. Tennekes and J. Lumley, A First Course in Turbulence, MIT Press, Cambridge, Mass., 1990

[33] H. Weber and P. Minnhagen, Monte-Carlo determination of the critical temperature for the twodimensional XY model, Phys. Rev. B 37, 5986-5989 (1988)

[34] M. V. Zagarola, A. J. Smits, S. A. Orszag, and V. Yakhot, Experiments in high Reynolds number turbulent pipe flow, AIAA paper 96-0654, Reno, Nevada, 1996 